\title{
Biologia de Psyllobora confluens alimentada com o fungo Erysiphe cichoracearum
}

\author{
Terezinha Monteiro dos Santos Cividanes ${ }^{(1)}$, Francisco Jorge Cividanes ${ }^{(2)}$ e Beatriz de Almeida de Matos $^{(3)}$
}

\begin{abstract}
(1)Agência Paulista de Tecnologia dos Agronegócios, Pólo Regional do Centro Leste, Av. Bandeirantes, no 2.419, CEP 14030-670 Ribeirão Preto, SP. E-mail: terezinha@aptaregional.sp.gov.br (2)Universidade Estadual Paulista, Fac. de Ciências Agrárias e Veterinárias, Dep. de Fitossanidade, Via de Acesso Prof. Paulo Donato Castellane, s/no, CEP 14884-900 Jaboticabal, SP. E-mail: fjcivida@fcav.unesp.br (3) Grupo Cosan, Unidade Gaza, Laboratório de Entomologia, Rua Amazonas, no 336, CEP 16900-000 Andradina, SP. E-mail: beatriz_almeidamatos@yahoo.com.br
\end{abstract}

Resumo - O objetivo deste trabalho foi avaliar os aspectos biológicos de Psyllobora confluens alimentada com o fungo Erysiphe cichoracearum em folhas de quiabeiro. Larvas recém-eclodidas de P. confluens foram individualizadas e alimentadas diariamente com hifas e conídios do fungo, em folhas de quiabeiro, a 23,6 63 e $27,4 \pm 2^{\circ} \mathrm{C}$ e fotófase de 12 horas. A duração dos estágios imaturos, os aspectos reprodutivos e a longevidade de P. confluens foram avaliados. O período de desenvolvimento de larva a adulto foi 20,5 dias a $23,6^{\circ} \mathrm{C}$ e 16,6 dias a $27,4^{\circ} \mathrm{C}$. Em ambas as temperaturas, $80 \%$ das larvas de $P$. confluens atingiram a fase adulta. Fêmeas de $P$. confluens apresentaram período de oviposição de 32,7 dias, com capacidade diária e total de oviposição de 16,8 e 439,9 ovos, respectivamente. A longevidade das fêmeas foi de 46,1 dias e a dos machos 58,7 dias. P. confluens, ao se alimentar do fungo E. cichoracearum em folhas de quiabeiro, completa o ciclo de vida e apresenta elevados padrões de sobrevivência e reprodução.

Termos para indexação: Coleoptera, Coccinellidae, Psylloborini, ciclo de vida, micofagia, oídio.

\section{Biology of Psyllobora confluens fed with Erysiphe cichoracearum fungus}

\begin{abstract}
The objective of this work was to assess the biological aspects of Psyllobora confluens fed with Erysiphe cichoracearum fungus in okra leaves. New hatched larvae of $P$. confluens were individualized and fed daily with powdery mildew hyphae and conidia of the fungus, in okra leaves, at $23.6 \pm 3$ and $27.4 \pm 3^{\circ} \mathrm{C}$ and 12 hours of photophase. The developmental period of immature stages, reproductive aspects and longevity of $P$. confluens were evaluated. The period from larvae development to adult emergence was 20.5 days at $23.6^{\circ} \mathrm{C}$ and 16.6 days at $27.4^{\circ} \mathrm{C}$. Under the tested temperatures, $80 \%$ of $P$. confluens larvae reached the adult phase. $P$. confluens females showed oviposition period of 32.7 days, with daily and total capacity of oviposition of 16.8 and 439.9 eggs, respectively. The longevity of females was 46.1 days and of males 58.7 days. P. confluens fed with E. cichoracearum in okra leaves completes its life cycle and presents high standard of survival and reproduction.
\end{abstract}

Index terms: Coleoptera, Coccinellidae, Psylloborini, life cycle, mycophagous habit, powdery mildew.

\section{Introdução}

Nas regiões tropicais, as doenças fúngicas são freqüentes e afetam principalmente a produção vegetal em campo e em casa de vegetação. O fungo Erysiphe cichoracearum De Candolle ocasiona a doença conhecida como oídio em culturas do quiabeiro, cucurbitáceas e plantas ornamentais. Esse patógeno coloniza ambas as faces das folhas da planta, forma inicialmente áreas esbranquiçadas irregulares e, posteriormente, ao recobrir toda a superfície, ocasiona um aspecto branco e pulverulento às folhas. Essa infestação provoca a desfolha das plantas, com conseqüente redução na produção (Massola Junior \& Bedendo, 1997; Bado \& Rodriguez, 1998; Soylu \& Yigit, 2002).

No controle dessa doença, é comum a aplicação de fungicidas químicos. No entanto, essa prática muitas vezes torna-se economicamente inviável e ineficiente, pois a aplicação freqüente desses produtos tem causado o surgimento de populações de fungos resistentes aos fungicidas (Sutherland, 2005).

Há uma tendência de os consumidores preferirem produtos saudáveis e sem resíduos de agroquímicos. Essa tendência, aliada a outros fatores, conduz ao desenvolvimento de pesquisas e tecnologias de controle biológico de patógenos (Soylu \& Yigit, 2002). 
Os coccinelídeos (Coleoptera: Coccinellidae) da tribo Psylloborini alimentam-se, tanto na fase larval quanto na adulta, de conídios e hifas de fungos fitopatogênicos que causam o oídio (Gordon, 1985). O gênero Psyllobora atua como agente de controle biológico de fungos em diversas culturas. Sutherland (2005) registrou a ocorrência de $P$. vigintimaculata taedata Say em 25 espécies de plantas de importância econômica. Soylu \& Yigit (2002) observaram que larvas e adultos da joaninha P. bisoctonotata (Mulsant), ao se alimentar de conídios do fungo E. cichoracearum, ocasionaram redução de $92 \%$ nas fontes de inóculo desse patógeno em folhas de quiabeiro. De acordo com determinações de Sutherland \& Parrella (2006), uma larva do coccinelídeo $P$. vigintimaculata foi capaz de eliminar todas as hifas e conídios do fungo E. cichoracearum, em área foliar de $6,3 \mathrm{~cm}^{2}$ da planta ornamental Zinnia elegans.

No Brasil, foi registrada a ocorrência de P. lenta (Mulsant) na cultura do quiabeiro, no Município de Guidoval, MG, e de P. confluens (Fabricius), que se alimentava de fungos do gênero Oidium, em cultivos de couve adjacentes a plantas de quiabeiro, em Seropédica, RJ (Leite et al., 2005; Resende et al., 2006). Alguns estudos referentes à determinação dos aspectos biológicos, reprodutivos e potencial na redução de fungos por espécies do gênero Psyllobora, foram realizados por Almeida \& Milleo (1998) no Brasil, Bado \& Rodriguez (1998) na Argentina, Soylu \& Yigit (2002) na Turquia, Sutherland (2005) e Sutherland \& Parrella (2006) nos Estados Unidos.

Apesar da importância de os coccinelídeos micófagos atuarem como agentes naturais no controle de fitopatógenos, ainda são poucas as pesquisas relacionadas a esses micopredadores.

O objetivo deste trabalho foi estudar a biologia de $P$. confluens alimentada com o fungo E. cichoracearum em folhas de quiabeiro.

\section{Material e Métodos}

O trabalho foi realizado no Laboratório de Entomologia, da Agência Paulista de Tecnologia dos Agronegócios, Unidade de Pesquisa e Desenvolvimento de Andradina, em Andradina, SP, de abril a agosto de 2006.

Adultos de $P$. confluens foram coletados em cultura do quiabeiro, transportados ao laboratório e mantidos em placas de Petri de $9 \mathrm{~cm}$ de diâmetro, para obtenção de ovos, coletados diariamente e transferidos para novas placas de Petri. Após eclosão, as larvas de $P$. confluens foram individualizadas em tubos de vidro de $8 \times 4 \mathrm{~cm}$, mantidos sob duas temperaturas, resultantes da média registrada nos dois períodos de realização do estudo sobre a fase imatura do coccinelídeo $(23,6 \pm 3$ e $27,4 \pm 2^{\circ} \mathrm{C}$ ), e fotófase de 12 horas. As larvas foram alimentadas diariamente ad libitum com estruturas vegetativas (micélio) e reprodutivas (conídios) do fungo E. cichoracearum, obtido de folhas de plantas de quiabeiro cultivadas em campo. Diariamente, foram avaliados os parâmetros: viabilidade dos ovos, período embrionário, duração dos estádios e duração dos períodos de larva, pré-pupa, pupa e larva-adulto.

O delineamento utilizado foi o inteiramente casualizado, com dois tratamentos constituídos pelas temperaturas e 24 repetições; os dados foram submetidos à análise de variância.

Com o auxílio de um microscópio estereoscópico, foram realizadas observações sobre o formato e a coloração dos ovos, de larvas, pupas e adultos de P. confluens. Avaliou-se o local de preferência de oviposição em cada seção de folha de quiabeiro oferecida ao coccinelídeo como substrato para a postura dos ovos.

Os padrões de fecundidade e de longevidade da fase adulta foram determinados a $23,6 \pm 3^{\circ} \mathrm{C}$. Após a emergência, adultos de P. confluens, originados de larvas alimentadas com o fungo E. cichoracearum, foram separados em dez casais e mantidos em placas de Petri de $9 \mathrm{~cm}$ de diâmetro x $2 \mathrm{~cm}$ de altura, vedadas na parte superior por filme de PVC. Diariamente, os casais foram alimentados com estruturas do fungo E. cichoracearum em seções de folhas de quiabeiro, e foram determinados os parâmetros biológicos: períodos de pré-oviposição, oviposição e pós-oviposição, capacidade diária e total de oviposição e longevidade.

O período embrionário e a viabilidade dos ovos de P. confluens foram determinados a $23,6^{\circ} \mathrm{C}$. Foram amostradas posturas, duas vezes por semana, das fêmeas dos dez casais de P. confluens, num total de 12 amostragens para cada fêmea.

\section{Resultados e Discussão}

Os ovos de P. confluens apresentaram formato elíptico, coloração bege, e foram depositados na face abaxial das folhas de quiabeiro, de maneira agrupada, próximo às áreas infectadas pelo fungo fitopatogênico (Figura 1 A). Sutherland (2005) observou que fêmeas 
de $P$. vigintimaculata taedata evitam realizar posturas em locais sem suprimento adequado de alimento para as larvas completarem o desenvolvimento. De acordo com Hodek (1973) e Iperti (1999), a maioria dos coccinelídeos realiza a postura dos ovos sob as folhas, próximo à fonte de alimento das larvas.

As larvas, pupas e adultos apresentaram coloração amarelo leitosa, com máculas castanho-escuras por toda a extensão dorsal do corpo (Figura 1 B, C e D). Tais observações estão de acordo com relatos de Bado \& Rodriguez (1998) e Sutherland (2005), que caracterizaram ovos, larvas e adultos de $P$. bicongregata (Boheman) e P. vigintimaculata taedata, respectivamente. Segundo Iperti (1999), coccinelídeos de hábito micófago geralmente apresentam coloração marrom-clara, branca ou amarelo-limão.
O período embrionário $(5,1 \pm 0,20$ dias $)$ e o porcentual de eclosão dos ovos $(87,2 \pm 2,39 \%)$ de $P$. confluens a $23,6^{\circ} \mathrm{C}$ foram similares aos observados por Bado \& Rodriguez (1998) e Sutherland (2005). Esses autores relataram que o período embrionário de $P$. bicongregata e $P$. vigintimaculata taedata durou 4 dias a $21^{\circ} \mathrm{C}$ e 4,7 dias a $25^{\circ} \mathrm{C}$, respectivamente. Sutherland (2005) obteve elevado porcentual de ovos férteis em $P$. vigintimaculata taedata.

Neste trabalho, as larvas de $P$. confluens apresentaram quatro estádios, dos quais apenas o desenvolvimento do quarto foi influenciado significativamente pela temperatura, tendo sido mais longo a $23,6^{\circ} \mathrm{C}$ (Tabela 1). Sutherland (2005), ao avaliar o desenvolvimento de $P$. vigintimaculata taedata alimentada com o fungo E. cichoracearum em folhas da planta ornamental
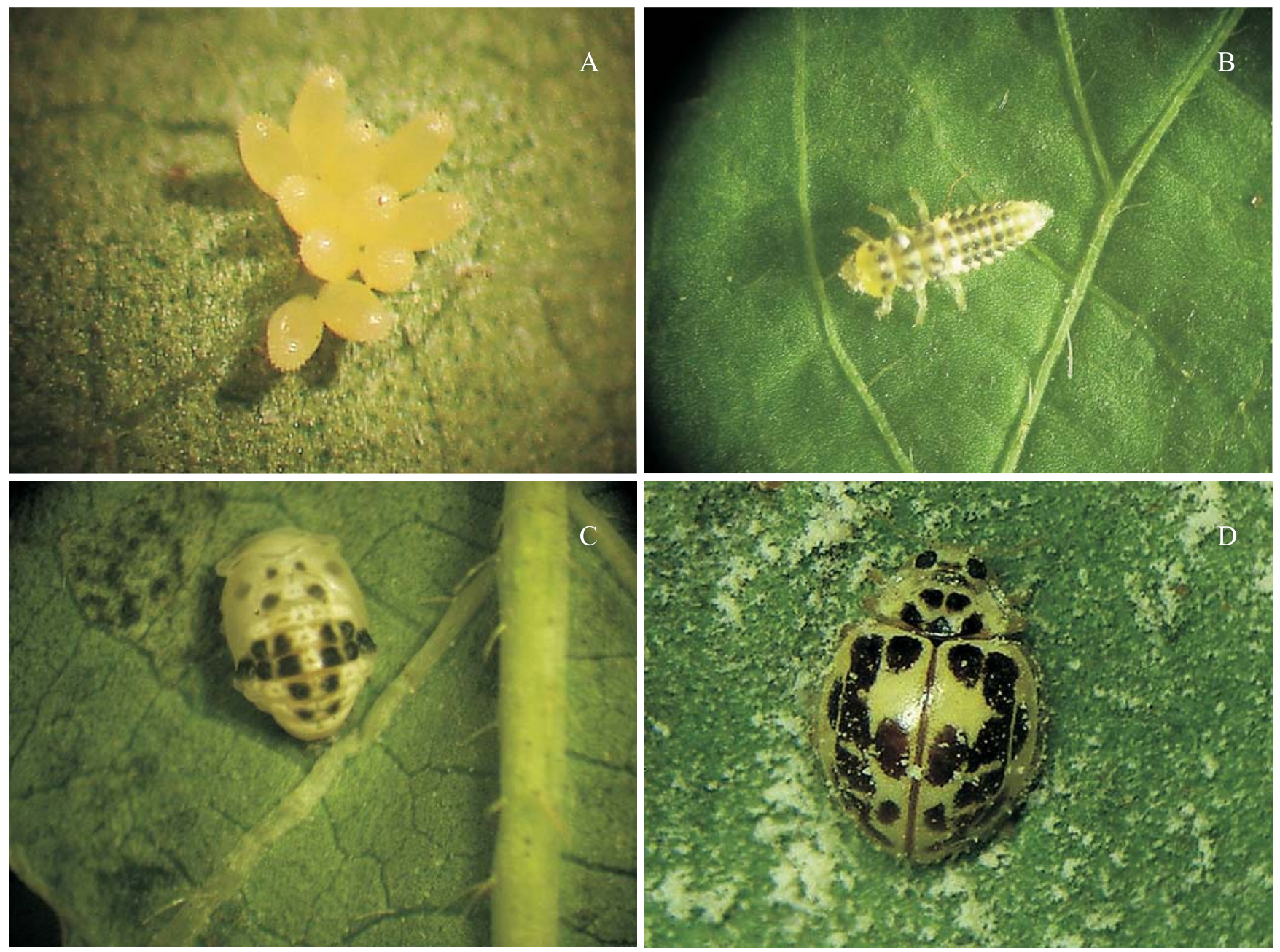

Figura 1. Fases de desenvolvimento de Psyllobora confluens. A: ovos; B: larva; C: pupa; D: adulto. 
Tabela 1. Duração (dias) das fases de desenvolvimento de Psyllobora confluens, alimentada com o fungo Erysiphe cichoracearum em folhas de quiabeiro, em duas temperaturas ${ }^{(1)}$.

\begin{tabular}{ccccccccc}
\hline Temperatura $\left({ }^{\circ} \mathrm{C}\right)$ & $1^{\circ}$ estádio & $2^{-}$estádio & $3^{-}$estádio & $4^{-}$estádio & Larva & Pré-pupa & Pupa & Larva-adulto \\
\hline 23,6 & $2,5 \pm 0,21 \mathrm{a}$ & $2,9 \pm 0,17 \mathrm{a}$ & $3,0 \pm 0,14 \mathrm{a}$ & $4,6 \pm 0,27 \mathrm{a}$ & $13,1 \pm 0,45 \mathrm{a}$ & $1,9 \pm 0,12 \mathrm{a}$ & $5,5 \pm 0,15 \mathrm{a}$ & $20,5 \pm 0,45 \mathrm{a}$ \\
27,4 & $2,7 \pm 0,24 \mathrm{a}$ & $2,5 \pm 0,15 \mathrm{a}$ & $2,9 \pm 0,22 \mathrm{a}$ & $3,6 \pm 0,29 \mathrm{~b}$ & $11,6 \pm 0,39 \mathrm{~b}$ & $1,8 \pm 0,10 \mathrm{~b}$ & $3,9 \pm 0,79 \mathrm{~b}$ & $16,6 \pm 0,53 \mathrm{~b}$ \\
\hline $\mathrm{CV}(\%)$ & 41,6 & 28,7 & 30,9 & 33,0 & 16,6 & 34,7 & 19,9 & 13,0 \\
\hline
\end{tabular}

(1)Médias ( \pm erro-padrão) seguidas por letras iguais não diferem entre si pelo teste $\mathrm{F}$, a $1 \%$ de probabilidade.

Z. elegans, observou que a $25^{\circ} \mathrm{C}$, o primeiro, segundo, terceiro e quarto estádios do coccinelídeo duraram 2,6, 2,6, 2,2 e 1,9 dias, respectivamente, enquanto a $20^{\circ} \mathrm{C}$, a duração desses foi prolongada para 4,3, 4,3, 3,6 e 3,2 dias.

A duração das fases de larva, pré-pupa, pupa e de larva-adulto de $P$. confluens diminuiu com o incremento térmico (Tabela 1). Sutherland (2005) constatou, para larvas de $P$. vigintimaculata taedata alimentada com o fungo E. cichoracearum, período larval de 15,4 dias a $20^{\circ} \mathrm{C}$ e 9,3 dias a $25^{\circ} \mathrm{C}$. Esse autor verificou, ainda, que a $20^{\circ} \mathrm{C}$ o período de desenvolvimento larval até a fase adulta do coccinelídeo durou 23,4 dias e decresceu para 14,4 dias a $25^{\circ} \mathrm{C}$. Iperti (1999) destacou que a duração do ciclo de vida dos coccinelídeos pode variar de acordo com o tamanho desses predadores, as condições térmicas e a especificidade trófica.

As larvas de $P$. confluens, alimentadas com E. cichoracearum e mantidas sob temperaturas de 23,6 e $27,4^{\circ} \mathrm{C}$, apresentaram $80 \%$ de sobrevivência até atingir a fase adulta. Essa baixa mortalidade, observada entre as fases de larva até a de pupa, indica que tanto a fonte de alimento quanto as temperaturas consideradas foram favoráveis ao desenvolvimento dessa espécie de micopredador.

A duração média do período de pré-oviposição ( $3,1 \pm 0,72$ dias) de $P$. confluens, observada neste trabalho, está de acordo com as observações de Iperti (1999) de que, para a maioria das espécies de coccinelídeos, o período de pré-oviposição atinge a duração máxima de sete dias. As fêmeas de P. confluens apresentaram período de oviposição médio

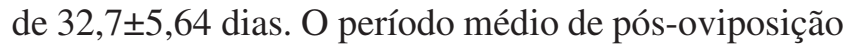
de $P$. confluens, observado neste trabalho (7,7 $\pm 2,13$ dias), é similar ao de várias espécies de coccinelídeos de hábito entomófago. A capacidade diária de oviposição e o total de ovos produzidos por fêmea

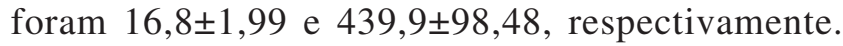
Segundo Quilici (1981), citado por Iperti (1999), o número total de ovos colocados por fêmeas de Coccinellidae varia de acordo com a espécie e pode, ainda, ser influenciado por fatores como temperatura, quantidade e qualidade de alimento disponível.

Os valores de longevidade de fêmeas e machos de $P$. confluens observados foram $46,1 \pm 6,10$ e $58,7 \pm 7,77$ dias, respectivamente. Hodek (1973) e Iperti (1999) ressaltaram que a longevidade dos coccinelídeos pode variar de alguns meses a até três anos.

\section{Conclusão}

Psyllobora confluens completa o ciclo de vida e apresenta elevados padrões de sobrevivência e de reprodução ao se alimentar do fungo Erysiphe cichoracearum em folhas de quiabeiro.

\section{Agradecimentos}

À Dra. Lúcia Massutti de Almeida, da Universidade Federal do Paraná, pela identificação do coccinelídeo; à equipe da Agência Paulista de Tecnologia dos Agronegócios, Unidade de Pesquisa e Desenvolvimento de Andradina, SP, pelo auxílio nas atividades de campo.

\section{Referências}

ALMEIDA, L.M.; MILLEO, J. The immature stages of Psyllobora gratiosa Mader, 1958 (Coleoptera: Coccinellidae) with some biological aspects. Journal of the New York Entomological Society, v.106, p.170-176, 1998.

BADO, S.G.; RODRIGUEZ, S.M. Aspectos morfológicos y biológicos de una vaquita micetófaga: Psyllobora bicongregata (Boh.) (Coleoptera: Coccinellidae). Revista de la Facultad de Agronomía, v.18, p.181-184, 1998.

GORDON, R.D. The Coccinellidae (Coleoptera) of America North of Mexico. Journal of the New York Entomological Society, v.93, p.1-912, 1985.

HODEK, I. Life history and biological properties. In: HODEK, I. (Ed.). Biology of Coccinellidae. The Hague: W. Junk N.V., 1973. p.70-75. 
IPERTI, G. Biodiversity of predaceous coccinellidae in relation to bioindication and economic importance. Agriculture, Ecosystems and Environment, v.74, p.323-342, 1999.

LEITE, G.L.D.; PICANÇO, M.; JHAM, G.N.; MOREIRA, M.D. Whitefly population dynamics in okra plantations. Pesquisa Agropecuária Brasileira, v.40, p.19-25, 2005.

MASSOLA JUNIOR, N.S.; BEDENDO, I.P. Doenças do quiabeiro. In: KIMATI, H.; AMORIM, L.; BERGAMIN FILHO, A.; CAMARGO, L.E.A.; REZENDE, J.A.M. (Ed.). Manual de Fitopatologia: doenças das plantas cultivadas. São Paulo: Agronômica Ceres, 1997. p.616-620.

RESENDE, A.L.S.; SILVA, E.E.; SILVA, V.B.; RIBEIRO, R.L.D.; GUERRA, J.G.M.; AGUIAR-MENEZES, E.L. Primeiro registro de Lipaphis pseudobrassicae Davis (Hemiptera; Aphididae) e sua associação com insetos predadores, parasitóides e formigas em couve (Cruciferae) no Brasil. Neotropical Entomology, v.35, p.551-555, 2006.
SOYLU, S.; YIGIT, A. Feeding of mycophagous ladybird, Psyllobora bisoctonotata (Muls.) on powdery mildew infested plants. In: ELAD, Y.; KÖHL, K.; SHTIENBERG, D. (Ed.). INFLUENCE OF ABIOTIC AND BIOTIC FACTORS ON BIOCONTROL AGENTS AT PINE BAY, 7., 2002, Kusadasi. Proceedings. Kusadasi: IOBC-WPRS, 2002. p.183-186.

SUTHERLAND, A.M. Evaluation of Psyllobora vigintimaculata (Say) (Coleoptera: Coccinellidae) for biological control of powdery mildew fungi (Erysiphales). 2005. 46p. Thesis (M.Sc.) - University of California, Davis.

SUTHERLAND, A.M.; PARRELLA, M.P. Quantification of powdery mildew consumption by a native coccinellid: implications for biological control? In: HODDLE, M.S.; JOHNSON, M.W. (Ed.). CALIFORNIA CONFERENCE ON BIOLOGICAL CONTROL, 5., 2006, Riverside. 2006. Proceedings. Riverside: University of California, 2006. p.188-192.

Recebido em 20 de junho de 2007 e aprovado em 30 de outubro de 2007 